\title{
Testing Weak Cross-Sectional Dependence in Large Panels
}

\author{
M. Hashem Pesaran
}

January 2012

CWPE 1208 


\title{
Testing Weak Cross-Sectional Dependence in Large Panels
}

\author{
M. Hashem Pesaran* \\ University of Cambridge \& University of Southern California
}

January 2012

\begin{abstract}
This paper considers testing the hypothesis that errors in a panel data model are weakly cross sectionally dependent, using the exponent of cross-sectional dependence $\alpha$, introduced recently in Bailey, Kapetanios and Pesaran (2012). It is shown that the implicit null of the $C D$ test depends on the relative expansion rates of $N$ and $T$. When $T=O\left(N^{\epsilon}\right)$, for some $0<\epsilon \leq 1$, then the implicit null of the $C D$ test is given by $0 \leq \alpha<(2-\epsilon) / 4$, which gives $0 \leq \alpha<1 / 4$, when $N$ and $T$ tend to infinity at the same rate such that $T / N \rightarrow \kappa$, with $\kappa$ being a finite positive constant. It is argued that in the case of large $N$ panels, the null of weak dependence is more appropriate than the null of independence which could be quite restrictive for large panels. Using Monte Carlo experiments, it is shown that the $C D$ test has the correct size for values of $\alpha$ in the range [0,1/4], for all combinations of $N$ and $T$, and irrespective of whether the panel contains lagged values of the dependent variables, so long as there are no major asymmetries in the error distribution.
\end{abstract}

Keywords: Exponent of cross-sectional dependence, Diagnostic tests, Panel data models, Dynamic heterogenous panels.

JEL-Classification: C12, C13, C33.

${ }^{*}$ This paper complements an earlier unpublished paper entitled "General Diagnostic Tests for Cross Section Dependence in Panels", which was distributed in 2004 as the Working Paper No. 0435 in Cambridge Working Papers in Economics, Faculty of Economics, University of Cambridge. I am grateful to Natalia Bailey and Majid Al-Sadoon for providing me with excellent research assistance, and for carrying out the Monte Carlo simulations. I would also like to thank George Kapetanios, Ron Smith, Takashi Yamagata, and Aman Ullah for helpful comments and discussions. Financial support from the ESRC Grant ES/I031626/1 is gratefully acknowledged. 


\section{Introduction}

This paper is concerned with tests of error dependence in the case of large linear regression panels where $N$ (the cross section dimension) is large. In the case of panels where $N$ is small (say 10 or less) and the time dimension of the panel $(T)$ is sufficiently large the cross correlations of the errors can be modelled (and tested statistically) using the seemingly unrelated regression equation (SURE) framework originally developed by Zellner (1962). In such panels where $N$ is fixed as $T \rightarrow \infty$, traditional time series techniques, including log-likelihood ratio tests, can be applied. A simple example of such a test is the Lagrange multiplier (LM) test of Breusch and Pagan (1980) which is based on the average of the squared pair-wise correlation coefficients of the residuals. However, in cases where $N$ is large standard techniques will not be applicable and other approaches must be considered.

In the literature on spatial statistics the extent of cross-sectional dependence is measured with respect to a given "connection or spatial matrix" that characterizes the pattern of spatial dependence according to a pre-specified set of rules. For example, the $(i, j)$ elements of a connection matrix, $w_{i j}$, could be set equal to 1 if the $i^{\text {th }}$ and $j^{\text {th }}$ regions are joined, and zero otherwise. See Moran (1948) and further elaborations by Cliff and Ord (1973, 1981). More recent accounts and references can be found in Anselin (1988, 2001), and Haining (2003, Ch. 7). This approach, apart from being dependent on the choice of the spatial matrix, is not appropriate in many economic applications where space is not a natural metric and economic and sociopolitical factors could be more appropriate. ${ }^{1}$

In the absence of ordering, tests of cross-sectional independence in the case of large $N$ panels have been considered in Frees (1995), Pesaran (2004), Pesaran, Ullah and Yamagata (2008), Sarafidis, Yamagata, Robertson (2009), and Baltagi, Feng and Kao (2011). Recent surveys are provided by Moscone and Tosetti (2009), and Sarafidis and Wansbeek (2012). The null hypothesis of these tests is the cross-sectional independence of the errors in the panel regressions, and the tests are based on pair-wise correlation coefficients of the residuals, $\hat{\rho}_{i j}$, for the $(i, j)$ units, computed assuming homogeneous or heterogeneous slopes.

The original LM test of Breusch and Pagan (1980), and its modified version for large $N$ panels by Pesaran, Ullah and Yamagata (2008), are based on $\hat{\rho}_{i j}^{2}$, and test the hypothesis that all pair-wise error covariances, $E\left(u_{i t}, u_{j t}\right)$, are equal to zero for $i \neq j$. In contrast, we show that the implicit null of the CD test, proposed in Pesaran (2004), which is based on $\hat{\rho}_{i j}$, is weak cross-sectional dependence discussed in Chudik, Pesaran and Tosetti (2011), and further developed in Bailey, Kapetanios and Pesaran (2012, BKP). More specifically, we show that the implicit null of the $C D$ test depends on the relative expansion rates of $N$ and $T$. In general, if $T=O\left(N^{\epsilon}\right)$ for some $\epsilon$ in the range $(0,1]$, then the implicit null of the $C D$ test is given by $0 \leq \alpha<(2-\epsilon) / 4$, where $\alpha$ is the exponent of cross-sectional dependence defined by $\bar{\rho}_{N}=\left[2 /(N(N-1)] \sum_{i=1}^{N-1} \sum_{j=i+1}^{N} \rho_{i j}=O\left(N^{2 \alpha-2}\right)\right.$, with $\rho_{i j}$ denoting the population correlation coefficient of $u_{i t}$ and $u_{j t}$. BKP show that $\alpha$ is identified and can be estimated consistently if $1 / 2<\alpha \leq 1$. This paper complements BKP by showing that the null hypothesis that $\alpha$ lies in the range $[0,1 / 2)$ can be tested using the $C D$ statistic if $\epsilon$ is close to zero ( $T$ almost fixed as $N \rightarrow \infty$ ), but in the case where $\epsilon=1$ ( $N$ and $T \rightarrow \infty$ at the same rate) then the implicit null of the $C D$ test is given by $\alpha<1 / 4$.

The null of weak cross-sectional dependence also seems more appropriate than the null of crosssectional independence in the case of large panel data models where only pervasive cross dependence

\footnotetext{
${ }^{1}$ For empirical applications where economic distance such as trade patterns are used in modelling of spatial correlations see Conley and Topa (2002) and Pesaran, Schuermann, and Weiner (2004).
} 
is of concern. For example, in portfolio analysis full diversification of idiosyncratic errors is achieved even if the errors are weakly correlated, and cross-sectional error independence is not required. (e.g. Chamberlain, 1983). In estimation of panels only strong cross-sectional error dependence can pose real problems, and in most applications weak cross-sectional error dependence does not pose serious estimation and inferential problems.

The small sample properties of the $C D$ test for different values of $\alpha$ and sample sizes are investigated by means of a number of Monte Carlo experiments. It is shown that the $C D$ test has the correct size for values of $\alpha$ in the range [0,1/4], for all combinations of $N$ and $T$, and irrespective of whether the panel contains lagged values of the dependent variables, so long as there are not major asymmetries in the error distributions. This is in contrast to the LM based tests (such as the one proposed by Pesaran, Ullah and Yamagata, 2008) that require the regressors to be strictly exogenous. In line with the theoretical results, the $C D$ test tends to over-reject if $T$ is large relative to $N$ and $\alpha$ is in the range $(1 / 4-1 / 2]$. The CD test also has satisfactory power for all values of $\alpha>1 / 2$ and rises with $N \sqrt{T}$ so long as $\alpha>1 / 2$.

The rest of the paper is organized as follows. The panel data model and the LM tests of error cross-sectional independence are introduced in Section 2. The concept of weak cross-sectional dependence is introduced and discussed in Section 3. The use of CD statistic for testing weak cross-sectional dependence is discussed in Section 4, where the asymptotic distribution of the test is rigorously established under the null of independence. The distribution of CD statistic under the more general null of weak dependence is considered in Section 5, and the conditions under which it tends to $N(0,1)$ are derived. The application of the test to heterogeneous dynamic panels is discussed in Section 6. Small sample evidence on the performance of the test is provided in Section 7. Section 8 concludes.

\section{Panel Data Models and the LM Type Tests of Cross- Sectional Error Independence}

Consider the following panel data model

$$
y_{i t}=\boldsymbol{\beta}_{i}^{\prime} \mathbf{x}_{i t}+u_{i t}, \text { for } i=1,2, \ldots, N ; t=1,2, \ldots, T,
$$

where $i$ indexes the cross section dimension and $t$ the time series dimension, $\mathbf{x}_{i t}$ is a $k \times 1$ vector of observed time-varying regressors (individual-specific as well as common regressors). An individualspecific intercept can be included by setting the first element of $\mathbf{x}_{i t}$ to unity. The coefficients, $\boldsymbol{\beta}_{i}$, are defined on a compact set and allowed to vary across $i$. For each $i, u_{i t} \sim \operatorname{IID}\left(0, \sigma_{i}^{2}\right)$, for all $t$, although they could be cross-sectionally correlated. ${ }^{2}$ The dependence of $u_{i t}$ across $i$ could arise in a number of different ways. It could be due to spatial dependence, omitted unobserved common components, or idiosyncractic pair-wise dependence of $u_{i t}$ and $u_{j t}(i \neq j)$ with no particular pattern of spatial or common components. The regressors could contain lagged values of $y_{i t}$, be either stationary (or integrated of order zero, $I(0)$ ) or have unit roots (or integrated of order 1, I(1)). But in the derivations below we assume $\mathbf{x}_{i t} \sim I(0)$, and distinguish between the static and dynamic cases where the regressors are strictly exogenous and when they are weakly exogenous, specifically when $\mathbf{x}_{i t}=\left(1, y_{i, t-1}, \ldots, y_{i, t-p}\right)$. The testing procedure is applicable to fixed and random effects models as well as to the more general heterogeneous slope or random coefficient specifications.

\footnotetext{
${ }^{2}$ The assumption that $u_{i t}$ 's are serially uncorrelated is not restrictive and can be accommodated by including a sufficient number of lagged values of $y_{i t}$ amongst the regressors.
} 


\subsection{LM Type Tests}

In the SURE context with $N$ fixed and $T \rightarrow \infty$, Breusch and Pagan (1980) proposed a Lagrange multiplier (LM) statistic for testing the null of zero cross equation error correlations which is particularly simple to compute and does not require the system estimation of the SURE model. The test is based on the following LM statistic

$$
C D_{l m}=T \sum_{i=1}^{N-1} \sum_{j=i+1}^{N} \hat{\rho}_{i j}^{2},
$$

where $\hat{\rho}_{i j}$ is the sample estimate of the pair-wise correlation of the residuals. Specifically,

$$
\hat{\rho}_{i j}=\hat{\rho}_{j i}=\frac{\sum_{t=1}^{T} e_{i t} e_{j t}}{\left(\sum_{t=1}^{T} e_{i t}^{2}\right)^{1 / 2}\left(\sum_{t=1}^{T} e_{j t}^{2}\right)^{1 / 2}},
$$

and $e_{i t}$ is the Ordinary Least Squares (OLS) estimate of $u_{i t}$ defined by

$$
e_{i t}=y_{i t}-\hat{\boldsymbol{\beta}}_{i}^{\prime} \mathbf{x}_{i t}
$$

with $\hat{\boldsymbol{\beta}}_{i}$ being the OLS estimator of $\boldsymbol{\beta}_{i}$ computed using the regression of $y_{i t}$ on $\mathbf{x}_{i t}$ for each $i$, separately. The LM test is valid for $N$ relatively small and $T$ sufficiently large. In this setting Breusch and Pagan show that under the null hypothesis of no cross-sectional dependence, specified by

$$
\operatorname{Cov}\left(u_{i t}, u_{j t}\right)=0, \text { for all } t, i \neq j
$$

$C D_{l m}$ is asymptotically distributed as chi-squared with $N(N-1) / 2$ degrees of freedom. As it stands this test is not applicable when $N \rightarrow \infty$. However, noting that under $H_{0}, T \hat{\rho}_{i j}^{2} \stackrel{a}{\sim} \chi_{1}^{2}$ with $\hat{\rho}_{i j}^{2}, i=1,2, . ., N-1, j=i+1,2, \ldots, N$, being asymptotically independent, the following scaled version of $C D_{l m}$ can be considered for testing the hypothesis of cross dependence even for $N$ and $T$ large:

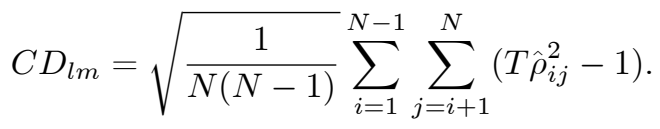

It is now easily seen that under $H_{0}$ with $T \rightarrow \infty$ first followed by $N \rightarrow \infty$ we would have $C D_{l m} \stackrel{a}{\sim}$ $N(0,1)$. However, this test is likely to exhibit substantial size distortions for $N$ large and $T$ small, a situation that can frequently arise in empirical applications. This is primarily due to the fact that for a finite $T, E\left(T \hat{\rho}_{i j}^{2}-1\right)$ will not be correctly centered at zero, and with $N$ large the incorrect centering of the LM statistic is likely to be accentuated, resulting in size distortions that tend to get worse with $N$. A bias corrected version of $C D_{l m}$ is proposed in Pesaran, Ullah and Yamagata (2008) under the assumptions that the regressors are strongly exogenous and the errors are normally distributed. In what follows we propose a test of weak cross-sectional dependence, which we argue to be more appropriate for large panels, where mere incidence of isolated dependencies are of little consequence for estimation or inference. 


\section{Weak Error Cross-Sectional Dependence}

As noted in the introduction when $N$ is large it is often more appropriate to consider the extent of error cross-sectional dependence rather than the extreme null hypothesis of error independence that underlies the LM type tests. This is in line, for example, with the assumption of approximate factor models discussed in Chamberlain (1983) in the context of capital asset pricing models. To this end we consider the following factor model for the errors

$$
u_{i t}=\omega_{i}\left(\gamma_{i}^{\prime} \mathbf{f}_{t}+\varepsilon_{i t}\right)
$$

where $\mathbf{f}_{t}=\left(f_{1 t}, f_{2 t}, \ldots, f_{m t}\right)^{\prime}$ is the $m \times 1$ vector of unobserved common factors ( $m$ being fixed) with $E\left(\mathbf{f}_{t}\right)=\mathbf{0}$, and $\operatorname{Cov}\left(\mathbf{f}_{t}\right)=\mathbf{I}_{m}, \gamma_{i}=\left(\gamma_{i 1}, \gamma_{i 2}, \ldots, \gamma_{i m}\right)^{\prime}$ is the associated vector of factor loadings, and $\varepsilon_{i t}$ are idiosyncratic errors that are cross-sectionally and serially independent with a unit variance, namely $\varepsilon_{i t} \sim I I D(0,1)$. The degree of cross-sectional dependence of the errors, $u_{i t}$, is governed by the rate at which the average pair-wise error correlation coefficient, $\bar{\rho}_{N}=$ $[2 / N(N-1)] \sum_{i=1}^{N-1} \sum_{j=i+1}^{N} \rho_{i j}$, tends to zero in $N$, where $\rho_{i j}=\operatorname{Corr}\left(u_{i t}, u_{j t}\right)$. In the case of the above factor model we have, $\operatorname{Var}\left(u_{i t}\right)=\sigma_{i}^{2}=\omega_{i}^{2}\left(1+\gamma_{i}^{\prime} \gamma_{i}\right), \rho_{i j}=\boldsymbol{\delta}_{i}^{\prime} \boldsymbol{\delta}_{j}$, for $i \neq j$, where

$$
\boldsymbol{\delta}_{i}=\frac{\gamma_{i}}{\sqrt{1+\gamma_{i}^{\prime} \gamma_{i}}}
$$

Then it is easily seen that

$$
\bar{\rho}_{N}=\left(\frac{N}{N-1}\right)\left(\overline{\boldsymbol{\delta}}_{N}^{\prime} \overline{\boldsymbol{\delta}}_{N}-\frac{\sum_{i=1}^{N} \boldsymbol{\delta}_{i}^{\prime} \boldsymbol{\delta}_{i}}{N^{2}}\right),
$$

where $\overline{\boldsymbol{\delta}}_{N}=N^{-1} \sum_{i=1}^{N} \boldsymbol{\delta}_{i}$.

Consider now the effects of the $j^{\text {th }}$ factor, $f_{j t}$, on the $i^{t h}$ error, $u_{i t}$, as measured by $\gamma_{i j}$, and suppose that these factor loadings take non-zero values for $M_{j}$ out of the $N$ cross-section units under consideration. Then following BKP, the degree of cross-sectional dependence due to the $j^{\text {th }}$ factor can be measured by $\alpha_{j}=\ln \left(M_{j}\right) / \ln (N)$, and the overall degree of cross-sectional dependence of the errors by $\alpha=\max _{j}\left(\alpha_{j}\right) . \alpha$ is the exponent of $N$ that gives the maximum number of errors, $M=\max _{j}\left(M_{j}\right)$, that are pair-wise correlated. The remaining $N-M$ units will only be partially correlated. BKP refer to $\alpha$ as the exponent of cross-sectional dependence. $\alpha$ can take any value in the range 0 to 1 , with 1 indicating the highest degree of cross-sectional dependence. Considering that $\gamma_{i}^{\prime} \gamma_{i}=O(m)$ where $m$ is fixed as $N \rightarrow \infty$, the exponent of cross-sectional dependence of the errors can be equivalently defined in terms of the scaled factor loadings, $\boldsymbol{\delta}_{i}=\left(\delta_{i 1}, \delta_{i 2}, \ldots, \delta_{i m}\right)^{\prime}$. Without loss of generality, suppose that only the first $M_{j}$ elements of $\delta_{i j}$ over $i$ are non-zero, and note that $^{3}$

$$
\bar{\delta}_{j, N}=\frac{1}{N}\left(\sum_{i=1}^{M_{j}} \delta_{i j}+\sum_{i=M_{j}+1}^{N} \delta_{i j}\right)=\frac{M_{i}}{N}\left(M_{j}^{-1} \sum_{i=1}^{M_{j}} \delta_{i j}\right)=N^{\alpha_{j}-1} \mu_{j}=O\left(N^{\alpha_{j}-1}\right),
$$

\footnotetext{
${ }^{3}$ The main results in the paper remain valid even if $\sum_{i=M_{j}+1}^{N} \delta_{i j}=O(1)$. But for expositional simplicity we maintain the assumption that $\sum_{i=M_{j}+1}^{N} \delta_{i j}=0$.
} 
where $\mu_{j}=\left(M_{j}^{-1} \sum_{i=1}^{M_{j}} \delta_{i j}\right) \neq 0$, for a finite $M_{j}$ and as $M_{j} \rightarrow \infty$. Similarly, $N^{-2} \sum_{i=1}^{N} \delta_{i j}^{\prime} \delta_{i j}=$ $O\left(N^{\alpha_{j}-2}\right)$, and using (8) we have

$$
\bar{\rho}_{N}=O\left(N^{2 \alpha-2}\right) .
$$

In what follows we develop a test of the null hypothesis that $\alpha<1 / 2$. The case where $\alpha>1 / 2$ is covered in BKP. The values of $\alpha$ in the range $[0,1 / 2)$ correspond to different degrees of weak cross-sectional dependence, as compared to values of $\alpha$ in the range $(1 / 2,1]$ that relate to different degrees of strong cross-sectional dependence.

\section{A Test of Weak Cross-Sectional Dependence}

Given that $\alpha$ is defined by the contraction rate of $\bar{\rho}_{N}$, we base the test of weak cross-sectional error dependence on its sample estimate, given by

$$
\widehat{\bar{\rho}}_{N}=\frac{2}{N(N-1)} \sum_{i=1}^{N-1} \sum_{j=i+1}^{N} \hat{\rho}_{i j},
$$

where $\hat{\rho}_{i j}$ is already defined by (2). The $C D$ test of Pesaran (2004) is in fact a scaled version of $\widehat{\bar{\rho}}_{N}$ which can be written as

$$
C D=\left[\frac{T N(N-1)}{2}\right]^{1 / 2} \widehat{\bar{\rho}}_{N} .
$$

In what follows we consider the distribution of the $C D$ statistic under three different null hypotheses. To establish comparability and some of the basic results we begin with $C D$ statistic under hypothesis of cross-sectional independence defined by

$$
H_{0}: \gamma_{i}=\mathbf{0}, \text { for all } i .
$$

We then consider the asymptotic distribution of the $C D$ statistic as $N$ and $T \rightarrow \infty$, such that $T=O\left(N^{\epsilon}\right)$, for $0<\epsilon \leq 1$, and show that the implicit null of the $C D$ test is given by

$$
H_{0}^{w}: \alpha<(2-\epsilon) / 4
$$

As argued earlier, such a null is much less restrictive for large $N$ panels than the pair-wise error independence assumption that underlies the $L M$ type tests which are based on $\hat{\rho}_{i j}^{2}$.

Initially, we derive the asymptotic distribution of the $C D$ test in the case of the standard panel data model, (1) subject to the following assumptions:

Assumption 1: The factor model, (6), holds. The idiosyncratic errors, $\varepsilon_{i t}$, are $I I D(0,1)$, are symmetrically distributed around 0 for all $i$ and $t, \mathbf{f}_{t} \sim \operatorname{IID}\left(\mathbf{0}, \mathbf{I}_{m}\right), \mathbf{f}_{t}$ and $\varepsilon_{i, t^{\prime}}$ are distributed independently, $0<\omega_{i}^{2}<K<\infty$. The factor loadings, $\gamma_{i}$, are independently distributed across $i$.

Assumption 2: The regressors, $\mathbf{x}_{i t}$, are strictly exogenous such that

$$
E\left(\varepsilon_{i t} \mid \mathbf{X}_{i}\right)=0 \text {, for all } i \text { and } t,
$$

where $\mathbf{X}_{i}=\left(\mathbf{x}_{i 1}, \mathbf{x}_{i 2}, \ldots, \mathbf{x}_{i T}\right)^{\prime}$, and

$$
E\left(\zeta_{i t}^{4}\right)<K<\infty,
$$


where $\zeta_{i t}=\varepsilon_{i t} /\left(T^{-1} \varepsilon_{i}^{\prime} \mathbf{M}_{i} \varepsilon_{i}\right)^{1 / 2}, \mathbf{M}_{i}=\mathbf{I}_{T}-\mathbf{X}_{i}\left(\mathbf{X}_{i}^{\prime} \mathbf{X}_{i}\right)^{-1} \mathbf{X}_{i}^{\prime}$, and $T^{-1} \mathbf{X}_{i}^{\prime} \mathbf{X}_{i}$ is a positive definite matrix for any fixed $T, T^{-1} \mathbf{X}_{i}^{\prime} \mathbf{X}_{i} \rightarrow p \boldsymbol{\Sigma}_{i i}$, as $T \rightarrow \infty$, with $\boldsymbol{\Sigma}_{i i}$ being a positive definite matrix. ${ }^{4}$

Assumption 3: $T>k+1$ and the OLS residuals, $e_{i t}$, defined by (3), are not all zero. ${ }^{5}$

Assumption 4: The factor loadings, $\gamma_{i}$, defined by (6) satisfy the $\alpha$-summability condition

$$
\sum_{i=1}^{N} \gamma_{i}=O\left(N^{\alpha}\right) .
$$

Theorem 1 Consider the regression model, (1), and suppose that Assumptions 1-3 hold, and the idiosyncratic errors, $\varepsilon_{i t}$, are symmetrically distributed around 0 , then under $H_{0}: \gamma_{i}=\mathbf{0}$, and for all $N>1$ and $T>k+1$ we have

$$
\begin{gathered}
E\left(\hat{\rho}_{i j}\right)=0, \text { for all } i \neq j, \\
E\left(\hat{\rho}_{i j} \hat{\rho}_{i s}\right)=0, \text { for all } i \neq j \neq s, \\
E(C D)=0, \\
\operatorname{Var}(C D)=1+\frac{T \bar{a}_{N}}{(T-k-1)^{2}}-\frac{(k+1)^{2}}{(T-k-1)^{2}}, \\
\bar{a}_{N}=\frac{2 \sum_{i=1}^{N-1} \sum_{j=i+1}^{N} \operatorname{Tr}\left(\mathbf{A}_{i} \mathbf{A}_{j}\right)}{N(N-1)}<k+1,
\end{gathered}
$$

where $\mathbf{A}_{i}=\mathbf{X}_{i}\left(\mathbf{X}_{i}^{\prime} \mathbf{X}_{i}\right)^{-1} \mathbf{X}_{i}^{\prime}, \hat{\rho}_{i j}$ and $C D$ are defined by (2) and (10), respectively. ${ }^{6}$

Proof:. First note that the pair-wise correlation coefficients can be written as

$$
\hat{\rho}_{i j}=T^{-1} \sum_{t=1}^{T} \xi_{i t} \xi_{j t}
$$

where $\xi_{i t}$ are the scaled residuals defined by

$$
\xi_{i t}=\frac{e_{i t}}{\left(T^{-1} \mathbf{e}_{i}^{\prime} \mathbf{e}_{i}\right)^{1 / 2}},
$$

$e_{i t}$ is the OLS residuals from the individual-specific regressions, defined by $(3)$, and $\mathbf{e}_{i}=\left(e_{i 1}, e_{i 2}, \ldots, e_{i T}\right)^{\prime}$. Also under $H_{0}, \mathbf{e}_{i}=\omega_{i} \mathbf{M}_{i} \varepsilon_{i}$, where $\varepsilon_{i}=\left(\varepsilon_{i 1}, \varepsilon_{i 2}, \ldots, \varepsilon_{i T}\right)^{\prime}$. Therefore, conditional on $\mathbf{x}_{i t}$, the scaled residuals, $\xi_{i t}$, are odd functions of the disturbances, $\varepsilon_{i t}$, and under Assumption 2 we have

$$
E\left(\xi_{i t} \mid \mathbf{X}_{i}\right)=0 \text {, for all } i \text { and } t .
$$

\footnotetext{
${ }^{4}$ The fourth-order moment of $\zeta_{i t}$ exists if $\sup _{i} E\left(\varepsilon_{i t}^{6}\right)<K<\infty$, and $T>k+4$. This result can be established using Lemmas in Lieberman (1994).

${ }^{5}$ The requirement $T>k+1$ can be relaxed under slope homogeneity assumption, $\boldsymbol{\beta}_{i}=\boldsymbol{\beta}$ where fixed effects residuals can be used in the construction of the CD statistic instead of $e_{i t}$.

${ }^{6}$ Similar results can also be obtained for fixed or random effects models. It suffices if the OLS residuals used in the computation of $\hat{\rho}_{i j}$ are replaced with associated residuals from fixed or random effects specifications. But the CD test based on the individual-specific OLS residuals are robust to slope and error-variance heterogeneity whilst the fixed or random effects residuals are not.
} 
Hence, unconditionally we also have

$$
E\left(\xi_{i t}\right)=0, \text { for all } i \text { and } t .
$$

Using this result in (21) now yields (recall that under $H_{0}$ the errors, $\varepsilon_{i t}$, are cross-sectionally independent),

$$
E\left(\hat{\rho}_{i j}\right)=0,
$$

which in turn establishes that (using (10))

$$
E(C D)=0,
$$

for any $N$, and all $T>k+1$. Under $H_{0}$ and Assumptions 1-3, $\hat{\rho}_{i j}$ and $\hat{\rho}_{i s}$ are cross-sectionally uncorrelated for $i, j$ and $s$, such that $i \neq j \neq s$. More specifically

$$
\begin{aligned}
E\left(\hat{\rho}_{i j} \hat{\rho}_{i s}\right) & =T^{-2} \sum_{t=1}^{T} \sum_{t^{\prime}=1}^{T} E\left(\xi_{i t} \xi_{j t} \xi_{i t^{\prime}} \xi_{s t^{\prime}}\right) \\
& =T^{-2} \sum_{t=1}^{T} \sum_{t^{\prime}=1}^{T} E\left(\xi_{i t} \xi_{i t^{\prime}}\right) E\left(\xi_{j t}\right) E\left(\xi_{s t^{\prime}}\right)=0, \text { for } i \neq j \neq s
\end{aligned}
$$

Also since the regressors are assumed to be strictly exogenous, we further have ${ }^{7}$

$$
\operatorname{Var}\left(\sqrt{T} \hat{\rho}_{i j}\right)=E\left(T \hat{\rho}_{i j}^{2}\right)=T \operatorname{Tr}\left(\mathbf{M}_{i} \mathbf{M}_{j}\right) /(T-k-1)^{2} .
$$

Using this result in (10) we have

$$
\begin{aligned}
\operatorname{Var}(C D) & =\frac{2 T}{N(N-1)(T-k-1)^{2}}\left(\sum_{i=1}^{N-1} \sum_{j=i+1}^{N}\left[T-2(k+1)+\operatorname{Tr}\left(\mathbf{A}_{i} \mathbf{A}_{j}\right)\right]\right) \\
& =\frac{T[T-2(k+1)]}{(T-k-1)^{2}}+\frac{2 T}{N(N-1)(T-k-1)^{2}}\left(\sum_{i=1}^{N-1} \sum_{j=i+1}^{N} \operatorname{Tr}\left(\mathbf{A}_{i} \mathbf{A}_{j}\right)\right)
\end{aligned}
$$

Hence

$$
\operatorname{Var}(C D)=1+\frac{T \bar{a}_{N}}{(T-k-1)^{2}}-\frac{(k+1)^{2}}{(T-k-1)^{2}}=1+O\left(\frac{1}{T}\right)
$$

where

$$
\bar{a}_{N}=\frac{2 \sum_{i=1}^{N-1} \sum_{j=i+1}^{N} \operatorname{Tr}\left(\mathbf{A}_{i} \mathbf{A}_{j}\right)}{N(N-1)} .
$$

But $\operatorname{Tr}\left(\mathbf{A}_{i} \mathbf{A}_{j}\right)<\left[\operatorname{Tr}\left(\mathbf{A}_{i}^{2}\right) \operatorname{Tr}\left(\mathbf{A}_{j}^{2}\right)\right]^{1 / 2}=k+1$, and we must also have $\bar{a}_{N}<k+1$. This completes the proof of the theorem.

The above results also suggest the following modified version of $C D$,

$$
\widetilde{C D}=\frac{C D}{\left[1+\frac{T \bar{a}_{N}}{(T-k-1)^{2}}-\frac{(k+1)^{2}}{(T-k-1)^{2}}\right]^{1 / 2}},
$$

\footnotetext{
${ }^{7}$ I am grateful to Aman Ullah for drawing my attention to this result. Also recall that $E\left(\hat{\rho}_{i j}\right)=0$.
} 
which is distributed exactly with a zero mean and a unit variance. In cases where $T-k-1$ is relatively large, and the regressors, $\mathbf{x}_{i t}$, are cross-sectionally weakly correlated, the term involving $\bar{a}_{N}$ in the expression for the variance of $C D$ will be small and both statistics are likely to perform very similarly, and the CD test is recommended on grounds of its simplicity. To keep the analysis simple, and without of loss generality, in what follows we shall focus on the $C D$ test.

\subsection{The distribution of the CD test under $H_{0}$}

Consider now the distribution of the $C D$ test. As shown in (17), the elements in the double summation that forms the CD statistic are uncorrelated but they need not be independently distributed when $T$ is finite. Therefore, when $T$ is finite the standard central limit theorems can not be exploited in order to derive the distribution of the $C D$ statistic. $^{8}$ To resolve the problem we first re-write the $C D$ statistic (defined by (10)) as

$$
C D_{N T}=\sqrt{\frac{2}{N(N-1)}}\left(\sum_{i=1}^{N-1} \sum_{j=i+1}^{N} \sqrt{T} \hat{\rho}_{i j}\right)
$$

and recall that $\hat{\rho}_{i j}=T^{-1} \sum_{t=1}^{T} \xi_{i t} \xi_{j t}$, where $\xi_{i t}$ is defined by (22). Now under $H_{0}: \gamma_{i}=0$, using standard results from regression analysis, we have

$$
\begin{gathered}
\xi_{i t}=\zeta_{i t}+T^{-1 / 2} h_{i t, T}, \\
h_{i t, T}=-\mathbf{x}_{i t}^{\prime}\left(\frac{\mathbf{X}_{i}^{\prime} \mathbf{X}_{i}}{T}\right)^{-1}\left(\frac{\mathbf{X}_{i}^{\prime} \boldsymbol{\zeta}_{i}}{\sqrt{T}}\right),
\end{gathered}
$$

where $\zeta_{i t}=\varepsilon_{i t} /\left(\varepsilon_{i}^{\prime} \mathbf{M}_{i} \varepsilon_{i} / T\right)^{1 / 2}$, and $\zeta_{i}=\left(\zeta_{i 1}, \zeta_{i 2}, \ldots, \zeta_{i T}\right)^{\prime}$. It will also prove helpful to note that under Assumptions 1 and 2, $E\left(\zeta_{i t}\right)=0, \operatorname{Cov}\left(\zeta_{i t}, \zeta_{j t}\right)=0$, for all $i \neq j$, and for each $i$, $\operatorname{Var}\left(\zeta_{i t}\right)=\sigma_{\zeta_{i}}^{2}=E\left(\frac{\varepsilon_{i t}^{2}}{T^{-1} \boldsymbol{\varepsilon}_{i}^{\prime} \mathbf{M}_{i} \varepsilon_{i}}\right)<K<\infty$. Furthermore, we have

$$
\begin{gathered}
E\left(h_{i t, T}\right)=0, \operatorname{Var}\left(h_{i t, T}\right)=\sigma_{\zeta_{i}}^{2} \mathbf{x}_{i t}^{\prime}\left(\frac{\mathbf{X}_{i}^{\prime} \mathbf{X}_{i}}{T}\right)^{-1} \mathbf{x}_{i t}<K<\infty \\
\operatorname{Cov}\left(h_{i t, T}, h_{j t, T}\right)=0, \text { and } \operatorname{Cov}\left(h_{i t, T}, \zeta_{i t}\right)=O\left(\frac{1}{\sqrt{T}}\right)
\end{gathered}
$$

Hence, for each $t$

$$
h_{t, N T}=N^{-1 / 2} \sum_{i=1}^{N} h_{i t, T}=O_{p}(1) ; \text { and } w_{t N}=N^{-1 / 2} \sum_{i=1}^{N} \zeta_{i t}=O_{p}(1) .
$$

With these preliminary results in mind, we write $C D_{N T}$ as

$$
C D_{N T}=\sqrt{\frac{2}{N(N-1)}} \sum_{i=1}^{N-1} \sum_{j=i+1}^{N} \frac{1}{\sqrt{T}} \sum_{t=1}^{T} \xi_{i t} \xi_{j t}
$$

\footnotetext{
${ }^{8}$ This corrects the statement made in error in Pesaran (2004).
} 
and note that

$$
\sum_{i=1}^{N-1} \sum_{j=i+1}^{N} \xi_{i t} \xi_{j t}=\frac{1}{2}\left[\left(\sum_{i=1}^{N} \xi_{i t}\right)^{2}-\sum_{i=1}^{N} \xi_{i t}^{2}\right]
$$

and hence

$$
C D_{N T}=\sqrt{\frac{N}{2(N-1)}} \frac{1}{\sqrt{T}} \sum_{t=1}^{T}\left[\left(\frac{\sum_{i=1}^{N} \xi_{i t}}{\sqrt{N}}\right)^{2}-\frac{\sum_{i=1}^{N} \xi_{i t}^{2}}{N}\right] .
$$

However, using (25),

$$
\begin{aligned}
\left(N^{-1 / 2} \sum_{i=1}^{N} \xi_{i t}\right)^{2}= & \left(N^{-1 / 2} \sum_{i=1}^{N} \zeta_{i t}\right)^{2}+\left((N T)^{-1 / 2} \sum_{i=1}^{N} h_{i t, T}\right)^{2} \\
& +2\left(N^{-1 / 2} \sum_{i=1}^{N} \zeta_{i t}\right)\left((N T)^{-1 / 2} \sum_{i=1}^{N} h_{i t, T}\right) \\
N^{-1} \sum_{i=1}^{N} \xi_{i t}^{2}= & N^{-1} \sum_{i=1}^{N} \zeta_{i t}^{2}+(N T)^{-1} \sum_{i=1}^{N} h_{i t, T}^{2}+ \\
& 2 T^{-1 / 2}\left(N^{-1 / 2} \sum_{i=1}^{N} h_{i t, T}\right)\left(N^{-1 / 2} \sum_{i=1}^{N} \zeta_{i t}\right) .
\end{aligned}
$$

Consider now the terms involving $h_{i t, T}$, and note that (using (27), (28), and (29))

$$
\begin{gathered}
\frac{1}{\sqrt{T}} \sum_{t=1}^{T}\left((N T)^{-1 / 2} \sum_{i=1}^{N} h_{i t, T}\right)^{2}=\frac{1}{\sqrt{T}} \frac{1}{T} \sum_{t=1}^{T}\left(N^{-1 / 2} \sum_{i=1}^{N} h_{i t, T}\right)^{2}=O_{p}\left(T^{-1 / 2}\right) \\
\frac{1}{\sqrt{T}} \frac{1}{N T} \sum_{t=1}^{T} \sum_{i=1}^{N} h_{i t, T}^{2}=O_{p}\left(T^{-1 / 2}\right) .
\end{gathered}
$$

Further,

$$
\frac{1}{\sqrt{T}} \sum_{t=1}^{T}\left(N^{-1 / 2} \sum_{i=1}^{N} \zeta_{i t}\right)\left((N T)^{-1 / 2} \sum_{i=1}^{N} h_{i t, T}\right)=\frac{1}{T} \sum_{t=1}^{T} w_{t N} h_{t, N T}
$$

where $w_{t N}$, and $h_{t, N T}$ are defined by (29), and are bounded independently distributed random variables with zero means, and $w_{t N}$ are serially uncorrelated. Hence

$$
\frac{1}{T} \sum_{t=1}^{T} w_{t N} h_{t, N T} \rightarrow p
$$

as $N$ and $T \rightarrow \infty$, in any order. Similarly,

$$
T^{-1 / 2}\left(N^{-1 / 2} \sum_{i=1}^{N} \zeta_{i t}\right)\left(N^{-1 / 2} \sum_{i=1}^{N} h_{i t, T}\right)=T^{-1 / 2} w_{t N} h_{t, N T}=O_{p}\left(T^{-1 / 2}\right) .
$$


Therefore,

$$
C D_{N T}=Z_{N T}+o_{p}(1)
$$

where

$$
Z_{N T}=\sqrt{\frac{N}{2(N-1)}} \frac{1}{\sqrt{T}} \sum_{t=1}^{T}\left[\left(\frac{\sum_{i=1}^{N} \zeta_{i t}}{\sqrt{N}}\right)^{2}-\frac{\sum_{i=1}^{N} \zeta_{i t}^{2}}{N}\right]
$$

and $o_{p}(1)$ indicates terms that tend to zero in probability as $N$ and $T \rightarrow \infty$, in any order.

To derive the distribution of $Z_{N T}$, recall that $w_{t N}=N^{-1 / 2} \sum_{i=1}^{N} \zeta_{i t}$, and write $Z_{N T}$ as

$$
Z_{N T}=\sqrt{\frac{N}{(N-1)}}\left(U_{N T}-V_{N T}\right)
$$

where

$$
U_{N T}=\frac{1}{\sqrt{T}} \sum_{t=1}^{T}\left(\frac{w_{t N}^{2}-E\left(w_{t N}^{2}\right)}{\sqrt{2}}\right)
$$

and

$$
V_{N T}=\frac{\sum_{t=1}^{T} \sum_{i=1}^{N}\left[\zeta_{i t}^{2}-E\left(\zeta_{i t}^{2}\right)\right]}{N \sqrt{2 T}}=\frac{\sum_{t=1}^{T} \sum_{i=1}^{N} \chi_{i t}}{N \sqrt{2 T}},
$$

where $\chi_{i t}=\zeta_{i t}^{2}-E\left(\zeta_{i t}^{2}\right)$, and $E\left(w_{t N}^{2}\right)=N^{-1} \sum_{i=1}^{N} E\left(\zeta_{i t}^{2}\right)$.

Under our assumptions, $\chi_{i t}$ are cross-sectionally and temporally independently distributed with mean 0 and a finite variance $\operatorname{Var}\left(\chi_{i t}\right)=E\left(\zeta_{i t}^{4}\right)-\sigma_{\zeta_{i}}^{4}$, such that $\sup _{i} \operatorname{Var}\left(\chi_{i t}\right)<K<\infty$. Hence, it readily follows that

$$
E\left(V_{N T}\right)=0, \operatorname{Var}\left(V_{N T}\right)=\frac{1}{2 T N^{2}} \sum_{t=1}^{T} \sum_{i=1}^{N} \operatorname{Var}\left(\chi_{i t}\right)<\frac{1}{2 N} \sup _{i} \operatorname{Var}\left(\chi_{i t}\right)=O\left(N^{-1}\right) .
$$

Consider now $U_{N T}$, and recall that $w_{t N}^{2}$ is temporally independent, with $E\left(w_{t N}\right)=0$, and $E\left(w_{t N}^{2}\right)=$ $N^{-1} \sum_{i=1}^{N} E\left(\zeta_{i t}^{2}\right)<\sup _{i} \sigma_{\zeta_{i}}^{2}<K<\infty$. Hence

$$
\begin{aligned}
E\left(U_{N T}\right) & =0 \\
\operatorname{Var}\left(U_{N T}\right) & =\frac{1}{2 T} \sum_{t=1}^{T} \operatorname{Var}\left(w_{t N}^{2}\right)=\frac{1}{2 T} \sum_{t=1}^{T}\left[E\left(w_{t N}^{4}\right)-\left[E\left(w_{t N}^{2}\right)\right]^{2}\right] .
\end{aligned}
$$

But, noting that $\zeta_{i t}$ are cross-sectionally independent,

$$
\begin{aligned}
E\left(w_{t N}^{4}\right) & =\frac{1}{N^{2}} \sum_{i=1}^{N} \sum_{j=1}^{N} \sum_{r=1}^{N} \sum_{s=1}^{N} E\left(\zeta_{i t} \zeta_{j t} \zeta_{r t} \zeta_{s t}\right) \\
& =\frac{3}{N^{2}}\left[\sum_{i=1}^{N} E\left(\zeta_{i t}^{2}\right)\right]^{2}+\frac{1}{N^{2}} \sum_{i=1}^{N} E\left(\zeta_{i t}^{4}\right) \\
& =3\left[E\left(w_{t N}^{2}\right)\right]^{2}+\frac{1}{N^{2}} \sum_{i=1}^{N} E\left(\zeta_{i t}^{4}\right),
\end{aligned}
$$


hence, substituting (38) into (37) we get

$$
\operatorname{Var}\left(U_{N T}\right)=\frac{1}{T} \sum_{t=1}^{T}\left[E\left(w_{t N}^{2}\right)\right]^{2}+\frac{1}{2 N^{2} T} \sum_{t=1}^{T} \sum_{i=1}^{N} E\left(\zeta_{i t}^{4}\right) .
$$

Now using (36) in (34), and then in (33), we have

$$
C D_{N T}=U_{N T}+O_{p}\left(N^{-1 / 2}\right)+o(1) .
$$

Also, noting that

$$
E\left(w_{t N}^{2}\right)=N^{-1} \sum_{i=1}^{N} E\left(\zeta_{i t}^{2}\right)=1+O\left(\frac{1}{N}\right)
$$

we obtain

$$
\begin{aligned}
U_{N T} & =\frac{1}{\sqrt{T}} \sum_{t=1}^{T}\left(\frac{w_{t N}^{2}-E\left(w_{t N}^{2}\right)}{\sqrt{2}}\right)=\frac{1}{\sqrt{T}} \sum_{t=1}^{T}\left(\frac{w_{t N}^{2}-1}{\sqrt{2}}\right)+ \\
w_{t N} & =N^{-1 / 2} \sum_{i=1}^{N} \zeta_{i t} .
\end{aligned}
$$

But for any $t$ and as $N \rightarrow \infty, w_{t N} \rightarrow_{d} N(0,1)$, and therefore $w_{t N}^{2} \rightarrow_{d} \chi_{t}^{2}(1)$, where $\chi_{t}^{2}(1)$, for $t=1,2, \ldots, T$ are independent chi-square variates with 1 degree of freedom. This in turn implies that as $N \rightarrow \infty, \frac{1}{\sqrt{2}}\left(w_{t N}^{2}-1\right)$, for $t=1,2, \ldots, T$, are independent random variates with mean zero and a unit variance. Hence, $U_{N T} \rightarrow_{d} N(0,1)$, as $N$ and $T \rightarrow \infty$, noting also that the term $O_{p}(\sqrt{T} / N)$ vanishes with $N \rightarrow \infty$, considering that $\sqrt{T} / N=O\left(N^{-1+\epsilon / 2}\right)$ and $\epsilon<1$.

Therefore, the $C D$ test is valid for $N$ and $T$ tending to infinity in any order. It is also clear that since the mean of $C D$ is exactly equal to zero for all fixed $T>k+1$ and $N$, the test is likely to have good small sample properties (for both $N$ and $T$ small), a conjecture which seems to be supported by extensive Monte Carlo experiments to be reported in Section 7.

\section{Asymptotic Distribution of the CD Test Under Weak Cross- Sectional Error Dependence}

In this section we consider the asymptotic distribution of the CD statistic under the null of weak cross-sectional dependence, $H_{0}^{w}$ defined by (12). To this end we assume that for each $i$

$$
\left\{\begin{array}{c}
T^{-1} \sum_{t=1}^{T} \varepsilon_{i t} \mathbf{f}_{t}=O_{p}\left(T^{-1 / 2}\right), T^{-1} \sum_{t=1}^{T} \mathbf{x}_{i t} \mathbf{f}_{t}^{\prime}=O_{p}\left(T^{-1 / 2}\right), \\
T^{-1} \sum_{t=1}^{T} \mathbf{f}_{t} \mathbf{f}_{t}^{\prime}=\mathbf{I}_{m}+O_{p}\left(T^{-1 / 2}\right) .
\end{array}\right.
$$

We also make the following standard assumptions about the regressors ${ }^{9}$

$$
\left(\frac{\mathbf{X}_{i}^{\prime} \mathbf{X}_{j}}{T}\right)=\boldsymbol{\Sigma}_{i j}+O_{p}\left(T^{-1 / 2}\right),\left(\frac{\mathbf{X}_{i}^{\prime} \varepsilon_{i}}{T}\right)=O_{p}\left(T^{-1 / 2}\right)
$$

\footnotetext{
${ }^{9}$ These assumptions allow for the inclusion of lagged dependent variables amongst the regressors and can be relaxed further to take account of non-stationary $I(1)$ regressors.
} 
where $\boldsymbol{\Sigma}_{i i}$ is a positive definite matrix.

Consider now the CD test statistic defined by (10) and note that under $H_{0}^{w}$, the vector of the OLS residuals is given by

$$
\mathbf{e}_{i}=\omega_{i}\left(\mathbf{M}_{i} \boldsymbol{\varepsilon}_{i}+\mathbf{M}_{i} \mathbf{F} \boldsymbol{\gamma}_{i}\right),
$$

where $\mathbf{F}=\left(\mathbf{f}_{1}, \mathbf{f}_{2}, \ldots, \mathbf{f}_{T}\right)^{\prime}$, and as before $\mathbf{M}_{i}=\mathbf{I}_{T}-\mathbf{X}_{i}\left(\mathbf{X}_{i}^{\prime} \mathbf{X}_{i}\right)^{-1} \mathbf{X}_{i}^{\prime}$. In this case the distribution of $\hat{\rho}_{i j}$ is quite complicated and depends on the magnitude of the factor loadings and the cross correlation patterns of the regressors and the unobserved factors. It does not, however, depend on the error variances, $\omega_{i}^{2}$. Under $H_{0}^{w}, \xi_{i t}$ defined by (22), can be written as

$$
\begin{aligned}
\xi_{i t}= & \frac{\boldsymbol{\gamma}_{i}^{\prime} \mathbf{f}_{t}+\varepsilon_{i t}}{\left(T^{-1} \varepsilon_{i}^{\prime} \mathbf{M}_{i} \varepsilon_{i}+2 T^{-1} \boldsymbol{\varepsilon}_{i}^{\prime} \mathbf{M}_{i} \mathbf{F} \boldsymbol{\gamma}_{i}+T^{-1} \boldsymbol{\gamma}_{i}^{\prime} \mathbf{F}^{\prime} \mathbf{M}_{i} \mathbf{F} \boldsymbol{\gamma}_{i}\right)^{1 / 2}} \\
& -\frac{\mathbf{x}_{i t}^{\prime}\left(\mathbf{X}_{i}^{\prime} \mathbf{X}_{i}\right)^{-1} \mathbf{X}_{i}^{\prime}\left(\mathbf{F} \boldsymbol{\gamma}_{i}+\boldsymbol{\varepsilon}_{i}\right)}{\left(T^{-1} \boldsymbol{\varepsilon}_{i}^{\prime} \mathbf{M}_{i} \varepsilon_{i}+2 T^{-1} \boldsymbol{\varepsilon}_{i}^{\prime} \mathbf{M}_{i} \mathbf{F} \boldsymbol{\gamma}_{i}+T^{-1} \boldsymbol{\gamma}_{i}^{\prime} \mathbf{F}^{\prime} \mathbf{M}_{i} \mathbf{F} \boldsymbol{\gamma}_{i}\right)^{1 / 2}},
\end{aligned}
$$

or more compactly

$$
\xi_{i t}=\tilde{\zeta}_{i t}+T^{-1 / 2} \tilde{h}_{i t, T}+\tilde{g}_{i t, T}
$$

where

$$
\begin{gathered}
\tilde{\zeta}_{i t}=\frac{\varepsilon_{i t}}{\psi_{i T}}, \tilde{h}_{i t, T}=-\mathbf{x}_{i t}^{\prime}\left(\frac{\mathbf{X}_{i}^{\prime} \mathbf{X}_{i}}{T}\right)^{-1}\left(\frac{\mathbf{X}_{i}^{\prime} \tilde{\boldsymbol{\zeta}}_{i}}{\sqrt{T}}\right), \\
\psi_{i T}=\left(T^{-1} \varepsilon_{i}^{\prime} \mathbf{M}_{i} \varepsilon_{i}+2 T^{-1} \boldsymbol{\varepsilon}_{i}^{\prime} \mathbf{M}_{i} \mathbf{F} \boldsymbol{\gamma}_{i}+T^{-1} \boldsymbol{\gamma}_{i}^{\prime} \mathbf{F}^{\prime} \mathbf{M}_{i} \mathbf{F} \boldsymbol{\gamma}_{i}\right)^{1 / 2},
\end{gathered}
$$

and

$$
\tilde{g}_{i t, T}=\frac{\boldsymbol{\gamma}_{i}^{\prime} \mathbf{f}_{t}-\mathbf{x}_{i t}^{\prime}\left(\mathbf{X}_{i}^{\prime} \mathbf{X}_{i}\right)^{-1} \mathbf{X}_{i}^{\prime} \mathbf{F} \gamma_{i}}{\psi_{i T}}
$$

Using (41) in (32), we now have

$$
C D_{N T}=\sqrt{\frac{N}{2(N-1)}} \frac{1}{\sqrt{T}} \sum_{t=1}^{T}\left[\begin{array}{c}
\left(\frac{\sum_{i=1}^{N} \tilde{\zeta}_{i t}+T^{-1 / 2} \tilde{h}_{i t, T}+\tilde{g}_{i t, T}}{\sqrt{N}}\right)^{2} \\
-\frac{\sum_{i=1}^{N}\left(\tilde{\zeta}_{i t}+T^{-1 / 2} \tilde{h}_{i t, T}+\tilde{g}_{i t, T}\right)^{2}}{N}
\end{array}\right] .
$$

Following the derivations in the previous section, it is possible to show that under Assumptions 1-4, (39), and (40), the null of weak cross-sectional dependence given by (12), then the $C D_{N T}$ statistics tends to $N(0,1)$ if

$$
\begin{gathered}
N^{-1} \sum_{i=1}^{N} E\left(\tilde{\zeta}_{i t}^{2}\right) \rightarrow 1 \\
\frac{1}{\sqrt{T}} \sum_{t=1}^{T}\left(\frac{\sum_{i=1}^{N} \tilde{g}_{i t, T}}{\sqrt{N}}\right)^{2} \rightarrow_{p} 0, \frac{1}{N \sqrt{T}} \sum_{t=1}^{T} \sum_{i=1}^{N} \tilde{g}_{i t, T}^{2} \rightarrow_{p} 0 .
\end{gathered}
$$

To establish these results, we first note that under Assumptions (39), and (40)

$$
\psi_{i T}^{2}=1+\gamma_{i}^{\prime} \gamma_{i}+O_{p}\left(T^{-1 / 2}\right)
$$


Using this result we have

$$
N^{-1} \sum_{i=1}^{N} E\left(\tilde{\zeta}_{i t}^{2}\right)=N^{-1} \sum_{i=1}^{N} E\left(\frac{\varepsilon_{i t}^{2}}{\psi_{i T}^{2}}\right) \rightarrow N^{-1} \sum_{i=1}^{N} \frac{1}{1+\gamma_{i}^{\prime} \gamma_{i}}=1-N^{-1} \sum_{i=1}^{N} \frac{\gamma_{i}^{\prime} \gamma_{i}}{1+\gamma_{i}^{\prime} \gamma_{i}} .
$$

But under (15), $\sum_{i=1}^{N} \frac{\boldsymbol{\gamma}_{i}^{\prime} \boldsymbol{\gamma}_{i}}{1+\boldsymbol{\gamma}_{i}^{\prime} \boldsymbol{\gamma}_{i}}=O\left(N^{\alpha}\right)$, and $N^{-1} \sum_{i=1}^{N} E\left(\tilde{\zeta}_{i t}^{2}\right) \rightarrow 1$, if $\alpha<1$.

Consider now the other two expressions in (45), and note that

$$
\begin{aligned}
\frac{1}{\sqrt{T}} \sum_{t=1}^{T}\left(\frac{\sum_{i=1}^{N} \tilde{g}_{i t, T}}{\sqrt{N}}\right)^{2}= & \frac{1}{\sqrt{T}} \sum_{t=1}^{T}\left(\frac{\left.\sum_{i=1}^{N} \tilde{\boldsymbol{\gamma}}_{i}^{\prime} \mathbf{f}_{t}-\sum_{i=1}^{N} \mathbf{x}_{i t}^{\prime}\left(\mathbf{X}_{i}^{\prime} \mathbf{X}_{i}\right)^{-1} \mathbf{X}_{i}^{\prime} \mathbf{F} \tilde{\gamma}_{i}\right)^{2}}{\sqrt{N}}=\frac{\sqrt{T}}{N}\left(\sum_{i=1}^{N} \tilde{\boldsymbol{\gamma}}_{i}^{\prime}\right)\left(\frac{\mathbf{F}^{\prime} \mathbf{F}}{T}\right)\left(\sum_{i=1}^{N} \tilde{\boldsymbol{\gamma}}_{i}\right)\right. \\
& +\frac{1}{N \sqrt{T}} \sum_{t=1}^{T}\left(\sum_{i=1}^{N} \mathbf{x}_{i t}^{\prime}\left(\mathbf{X}_{i}^{\prime} \mathbf{X}_{i}\right)^{-1} \mathbf{X}_{i}^{\prime} \mathbf{F} \tilde{\gamma}_{i}\right)^{2} \\
& -2 \frac{1}{N \sqrt{T}}\left(\sum_{i=1}^{N} \tilde{\gamma}_{i}^{\prime}\right) \sum_{i=1}^{N}\left(\mathbf{F}^{\prime} \mathbf{X}_{i}\right)\left(\mathbf{X}_{i}^{\prime} \mathbf{X}_{i}\right)^{-1}\left(\mathbf{X}_{i}^{\prime} \mathbf{F}\right) \tilde{\boldsymbol{\gamma}}_{i}
\end{aligned}
$$

where $\tilde{\gamma}_{i}=\tilde{\gamma}_{i} / \psi_{i T}$. But under (39) and (15) and setting $T=O\left(N^{\epsilon}\right)$, we have

$$
\frac{\sqrt{T}}{N}\left(\sum_{i=1}^{N} \tilde{\gamma}_{i}^{\prime}\right)\left(\frac{\mathbf{F}^{\prime} \mathbf{F}}{T}\right)\left(\sum_{i=1}^{N} \tilde{\gamma}_{i}\right)=O_{p}\left(N^{2 \alpha-1+\epsilon / 2}\right)
$$

and $\frac{\sqrt{T}}{N}\left(\sum_{i=1}^{N} \tilde{\gamma}_{i}^{\prime}\right)\left(\frac{\mathbf{F}^{\prime} \mathbf{F}}{T}\right)\left(\sum_{i=1}^{N} \tilde{\gamma}_{i}\right) \rightarrow_{p} 0$, as $N \rightarrow \infty$, if $2 \alpha-1+\epsilon / 2<0$, or if $\alpha<(2-\epsilon) / 4$. Similarly,

$$
\begin{aligned}
& \frac{1}{N \sqrt{T}} \sum_{t=1}^{T}\left(\sum_{i=1}^{N} \mathbf{x}_{i t}^{\prime}\left(\mathbf{X}_{i}^{\prime} \mathbf{X}_{i}\right)^{-1} \mathbf{X}_{i}^{\prime} \mathbf{F} \tilde{\boldsymbol{\gamma}}_{i}\right)^{2} \\
= & \frac{1}{N \sqrt{T}} \sum_{t=1}^{T} \sum_{i=1}^{N} \sum_{j=1}^{N} \tilde{\boldsymbol{\gamma}}_{i}^{\prime} \mathbf{F}^{\prime} \mathbf{X}_{i}\left(\mathbf{X}_{i}^{\prime} \mathbf{X}_{i}\right)^{-1} \mathbf{x}_{i t} \mathbf{x}_{j t}^{\prime}\left(\mathbf{X}_{j}^{\prime} \mathbf{X}_{j}\right)^{-1} \mathbf{X}_{j}^{\prime} \mathbf{F} \tilde{\boldsymbol{\gamma}}_{j} \\
= & \frac{1}{N \sqrt{T}} \sum_{i=1}^{N} \sum_{j=1}^{N} \tilde{\boldsymbol{\gamma}}_{i}^{\prime}\left(\mathbf{F}^{\prime} \mathbf{X}_{i}\right)\left(\mathbf{X}_{i}^{\prime} \mathbf{X}_{i}\right)^{-1}\left(\mathbf{X}_{i}^{\prime} \mathbf{X}_{j}\right)\left(\mathbf{X}_{j}^{\prime} \mathbf{X}_{j}\right)^{-1}\left(\mathbf{X}_{j}^{\prime} \mathbf{F}\right) \tilde{\boldsymbol{\gamma}}_{j} \\
= & \frac{1}{N \sqrt{T}}\left(\sum_{i=1}^{N} \tilde{\gamma}_{i}^{\prime} \mathbf{F}^{\prime} \mathbf{A}_{i}\right)\left(\sum_{i=1}^{N} \tilde{\boldsymbol{\gamma}}_{i}^{\prime} \mathbf{F}^{\prime} \mathbf{A}_{i}\right)^{\prime},
\end{aligned}
$$

where $\mathbf{A}_{i}=\mathbf{X}_{i}\left(\mathbf{X}_{i}^{\prime} \mathbf{X}_{i}\right)^{-1} \mathbf{X}_{i}$. But (using the norm $\|\mathbf{A}\|^{2}=\operatorname{Tr}\left(\mathbf{A}^{\prime} \mathbf{A}\right)$ )

$$
\left\|\sum_{i=1}^{N} \tilde{\gamma}_{i}^{\prime} \mathbf{F}^{\prime} \mathbf{A}_{i}\right\| \leq \sum_{i=1}^{N}\left\|\tilde{\gamma}_{i}^{\prime}\right\|\left\|\mathbf{F}^{\prime} \mathbf{A}_{i}\right\| \leq \sum_{i=1}^{N}\left(\tilde{\gamma}_{i}^{\prime} \tilde{\gamma}_{i}\right)^{1 / 2}\left[\operatorname{Tr}\left(\mathbf{F}^{\prime} \mathbf{A}_{i} \mathbf{F}\right)\right]^{1 / 2},
$$


and $\mathbf{F}^{\prime} \mathbf{A}_{i} \mathbf{F}=\left(\frac{\mathbf{F}^{\prime} \mathbf{X}_{i}}{\sqrt{T}}\right)\left(\frac{\mathbf{X}_{i}^{\prime} \mathbf{X}_{i}}{T}\right)^{-1}\left(\frac{\mathbf{X}_{i} \mathbf{F}}{\sqrt{T}}\right)=O_{p}(1)$, by Assumption (39). Hence $\sum_{i=1}^{N} \tilde{\gamma}_{i}^{\prime} \mathbf{F}^{\prime} \mathbf{A}_{i}=$ $O_{p}\left(\sum_{i=1}^{N}\left(\tilde{\gamma}_{i}^{\prime} \tilde{\gamma}_{i}\right)^{1 / 2}\right)=O_{p}\left(N^{\alpha}\right)$, and for $T=O\left(N^{\epsilon}\right),{ }^{10}$

$$
\frac{1}{N \sqrt{T}} \sum_{t=1}^{T}\left(\sum_{i=1}^{N} \mathbf{x}_{i t}^{\prime}\left(\mathbf{X}_{i}^{\prime} \mathbf{X}_{i}\right)^{-1} \mathbf{X}_{i}^{\prime} \mathbf{F} \tilde{\gamma}_{i}\right)^{2}=\frac{1}{N \sqrt{T}}\left(\sum_{i=1}^{N} \tilde{\gamma}_{i}^{\prime} \mathbf{F}^{\prime} \mathbf{A}_{i}\right)\left(\sum_{i=1}^{N} \tilde{\gamma}_{i}^{\prime} \mathbf{F}^{\prime} \mathbf{A}_{i}\right)^{\prime}=O\left(N^{2 \alpha-1-\epsilon / 2}\right)
$$

Thus, the second term of (47) vanishes if $\alpha<(2+\epsilon) / 4$, which is satisfied if $\alpha<(2-\epsilon) / 4$. It is also easily established that the third term in (46) also vanish if $\alpha<(2+\epsilon) / 4$.

Finally, consider the second expression in (45) and note that

$$
\begin{aligned}
& \frac{1}{N \sqrt{T}} \sum_{t=1}^{T} \sum_{i=1}^{N} \tilde{g}_{i t, T}^{2} \\
= & \frac{1}{N \sqrt{T}} \sum_{t=1}^{T} \sum_{i=1}^{N}\left[\tilde{\gamma}_{i}^{\prime} \mathbf{f}_{t}-\mathbf{x}_{i t}^{\prime}\left(\mathbf{X}_{i}^{\prime} \mathbf{X}_{i}\right)^{-1} \mathbf{X}_{i}^{\prime} \mathbf{F} \tilde{\gamma}_{i}\right]^{2}, \\
= & \frac{1}{N \sqrt{T}} \sum_{t=1}^{T} \sum_{i=1}^{N}\left[\begin{array}{c}
\tilde{\gamma}_{i}^{\prime} \mathbf{f}_{t} \mathbf{f}_{t}^{\prime} \tilde{\gamma}_{i}+\tilde{\boldsymbol{\gamma}}_{i}^{\prime} \mathbf{F}^{\prime} \mathbf{X}_{i}\left(\mathbf{X}_{i}^{\prime} \mathbf{X}_{i}\right)^{-1} \mathbf{x}_{i t} \mathbf{x}_{i t}^{\prime}\left(\mathbf{X}_{i}^{\prime} \mathbf{X}_{i}\right)^{-1} \mathbf{X}_{i}^{\prime} \mathbf{F} \tilde{\boldsymbol{\gamma}}_{i}^{\prime} \mathbf{f}_{t} \mathbf{x}_{i t}^{\prime}\left(\mathbf{X}_{i}^{\prime} \mathbf{X}_{i}\right)^{-1} \mathbf{X}_{i}^{\prime} \mathbf{F} \tilde{\gamma}_{i}
\end{array}\right] \\
= & \frac{1}{N \sqrt{T}} \sum_{i=1}^{N}\left(\tilde{\gamma}_{i}^{\prime} \mathbf{F}^{\prime} \mathbf{F} \tilde{\boldsymbol{\gamma}}_{i}-\tilde{\gamma}_{i}^{\prime} \mathbf{F}^{\prime} \mathbf{A}_{i} \mathbf{F} \tilde{\boldsymbol{\gamma}}_{i}\right) .
\end{aligned}
$$

Using similar lines of reasoning as above, it is easily established that $\frac{1}{N \sqrt{T}} \sum_{t=1}^{T} \sum_{i=1}^{N} \tilde{g}_{i t, T}^{2} \rightarrow{ }_{p} 0$, if $\alpha<(2-\epsilon) / 2$, which is satisfied if $\alpha<(2-\epsilon) / 4$, considering that $\epsilon \leq 1$.

The above results are summarized in the following theorem:

Theorem 2 Consider the panel data model (1), and suppose that Assumptions 1 to 4, (39), and (40) hold. Suppose further that $N$ and $T \rightarrow \infty$, such that $T / N^{\epsilon} \rightarrow \kappa$, where $\epsilon$ lies in the range $(0,1]$ and $\kappa$ is a finite positive non-zero constant. Then the CD statistic defined by (10) has the limiting $N(0,1)$ distribution as $N$ and $T \rightarrow \infty$, so long as $\alpha$, the exponent of cross- sectional dependence of the errors, $u_{i t}$, is less than $(2-\epsilon) / 4$. In the case where $N$ and $T$ tend to infinity at the same rate the $C D$ statistic tends to $N(0,1)$ if $\alpha<1 / 4$. The CD test is consistent for all values of $\alpha>1 / 2$, with the power of the test rising in $\alpha$ and $N \sqrt{T}$.

The power properties of the CD test follows directly from the derivations provided above.

\footnotetext{
${ }^{10}$ Note that since $\psi_{i T}>0$ then the order of $\sum_{i=1}^{N} \gamma_{i}$ and $\sum_{i=1}^{N} \tilde{\gamma}_{i}$ will be the same.
} 


\section{Cross Section Dependence in Heterogeneous Dynamic Pan- els}

The analyses of the previous sections readily extend to models with lagged dependent variables. As an example consider the following first-order dynamic panel data model

$$
y_{i t}=\mu_{i}\left(1-\lambda_{i}\right)+\lambda_{i} y_{i, t-1}+\sigma_{i} u_{i t}, i=1,2, \ldots, N ; t=1,2, \ldots, T,
$$

where $y_{i 0}=\mu_{i}+c_{i} \varepsilon_{i 0}$, and for each $i$ the errors, $u_{i t}, t=0,1, \ldots, T$ are serially uncorrelated with a zero mean and a unit variance but could be cross-sectionally correlated. The above specification is quite general and allows the underlying $\operatorname{AR}(1)$ processes to be stationary for some individuals and have a unit root for some other individuals in the panel. In the stationary case, if the process has started a long time in the past we would have $c_{i}=\sigma_{i}\left(1-\lambda_{i}\right)^{-1 / 2}$. In the unit root case where $\lambda_{i}=1, c_{i}$ could still differ across $i$ depending on the number of periods that the $i^{\text {th }}$ unit root process has been in operation before the initial observation, $y_{i 0}$.

Given the complicated nature of the dynamics and the mix of stationary and unit root processes that could prevail in a given panel, testing for cross-sectional dependence is likely to be complicated and in general might require $N$ and $T$ to be large. As it is well known the OLS estimates of $c_{i}$ and $\lambda_{i}$ for the individual series, as well as the fixed and random effects panel estimates used under slope homogeneity $\left(\lambda_{i}=\lambda\right)$ are biased when $T$ is small. The bias could be substantial for values of $\lambda_{i}$ near unity. Nevertheless, as it turns out in the case of pure autoregressive panels (without exogenous regressors) the $C D$ test is still valid for all values of $\lambda_{i}$ including those close to unity. The main reason lies in the fact that despite the small sample bias of the parameter estimates, the OLS or fixed effects residuals have exactly mean zero even for a fixed $T$, so long as $u_{i t} t=0,1, \ldots, T$ are symmetrically distributed. To see this we first write the individual AR(1) processes in matrix notations as

$$
\mathbf{\Lambda}_{i}\left(\mathbf{y}_{i}^{*}-\mu_{i} \boldsymbol{\tau}_{T+1}\right)=\mathbf{D}_{i} \mathbf{u}_{i}^{*},
$$

where $\mathbf{y}_{i}^{*}=\left(y_{i 0}, y_{i 1}, \ldots, y_{i T}\right)^{\prime}, \mathbf{u}_{i}^{*}=\left(u_{i 0}, u_{i 1}, \ldots, u_{i T}\right)^{\prime}, \boldsymbol{\tau}_{T+1}$ is a $(T+1) \times 1$ vector of ones, $\mathbf{D}_{i}$ is a $(T+1) \times(T+1)$ diagonal matrix with its first element equal to $\delta_{i}$ and the remaining elements equal to $\sigma_{i}$, and

$$
\boldsymbol{\Lambda}_{i}=\left(\begin{array}{cccccc}
1 & 0 & 0 & \cdots & 0 & 0 \\
-\lambda_{i} & 1 & 0 & & 0 & 0 \\
0 & -\lambda_{i} & 1 & & 0 & 0 \\
\vdots & \vdots & \vdots & \cdots & \vdots & \vdots \\
0 & 0 & 0 & \cdots & 1 & 0 \\
0 & 0 & 0 & \cdots & -\lambda_{i} & 1
\end{array}\right)
$$

The OLS estimates of individual intercepts and slopes can now be written as

$$
\begin{gathered}
\hat{\lambda}_{i}=\frac{\mathbf{u}_{i}^{* \prime} \mathbf{H}_{i}^{\prime} \mathbf{G}_{1}^{\prime} \mathbf{M}_{\tau} \mathbf{G}_{0} \mathbf{H}_{i} \mathbf{u}_{i}^{*}}{\mathbf{u}_{i}^{* \prime} \mathbf{H}_{i}^{\prime} \mathbf{G}_{1}^{\prime} \mathbf{M}_{\tau} \mathbf{G}_{1} \mathbf{H}_{i} \mathbf{u}_{i}^{*}} \\
\hat{\alpha}_{i}=\mu_{i}\left(1-\lambda_{i}\right)+\left(\frac{\boldsymbol{\tau}_{T}^{\prime} \mathbf{G}_{0} \mathbf{H}_{i} \mathbf{u}_{i}^{*}}{T}\right)-\left(\frac{\boldsymbol{\tau}_{T}^{\prime} \mathbf{G}_{1} \mathbf{H}_{i} \mathbf{u}_{i}^{*}}{T}\right) \hat{\lambda}_{i} .
\end{gathered}
$$


where $\mathbf{H}_{i}=\boldsymbol{\Lambda}_{i}^{-1} \mathbf{D}_{i}, \mathbf{G}_{0}=\left(\mathbf{0}_{T \times 1}, \mathbf{I}_{T}\right), \mathbf{G}_{1}=\left(\mathbf{I}_{T}, \mathbf{0}_{T \times 1}\right)$, and $\mathbf{0}_{T \times 1}$ is a $T \times 1$ vector of zeros. Using these results we now have the following expression for the OLS residuals, $e_{i t}=y_{i t}-\hat{\alpha}_{i}-\hat{\beta}_{i} y_{i, t-1}$,

$$
e_{i t}=-\left(\hat{\lambda}_{i}-\lambda_{i}\right)\left(y_{i, t-1}-\mu_{i}\right)+\sigma_{i} u_{i t}-\left(\frac{\boldsymbol{\tau}_{T}^{\prime} \mathbf{G}_{0} \mathbf{H}_{i} \mathbf{u}_{i}^{*}}{T}\right)+\left(\frac{\boldsymbol{\tau}_{T}^{\prime} \mathbf{G}_{1} \mathbf{H}_{i} \mathbf{u}_{i}^{*}}{T}\right) \hat{\lambda}_{i} .
$$

Using (48) we also note that $y_{i, t-1}-\mu_{i}=\mathbf{s}_{t-1}^{\prime} \mathbf{H}_{i} \mathbf{u}_{i}^{*}$, where $\mathbf{s}_{t-1}$ is a $(T+1) \times 1$ selection vector with zero elements except for its $t^{t h}$ element which is unity. Therefore, $e_{i t}$, and hence $\xi_{i t}=$ $\left(T^{-1} \mathbf{e}_{i}^{\prime} \mathbf{e}_{i}\right)^{-1 / 2} e_{i t}$ will be an odd function of $\mathbf{u}_{i}^{*}$, and we have $E\left(\xi_{i t}\right)=0, t=1,2, . ., T$, under the assumption that $\mathbf{u}_{i}^{*}$ has a symmetric distribution. Thus, under the null hypothesis that $u_{i t}$ and $u_{j t}$ are cross-sectionally independent we have $E\left(\hat{\rho}_{i j}\right)=0$, and the CD test continues to hold for pure dynamic heterogeneous panel data models. Under weak cross-sectionally dependent errors it is easily seen that the conditions (44) and (45) are satisfied under (12) as $N$ and $T \rightarrow \infty$. Finally, the $C D$ test will be robust to structural breaks so long as the unconditional mean of the process remains unchanged, namely if $E\left(y_{i t}\right)=\mu_{i}$, for all $t$. For proofs and further discussions see Pesaran $(2004) \cdot{ }^{11}$

\section{$7 \quad$ Small Sample Evidence}

In investigating the small sample properties of the $\mathrm{CD}$ test we consider two basic panel data regression models, a static model with a single exogenous regressor, and a dynamic second-order autoregressive specification. Both models allow for heterogeneity of slopes and error variances and include two unobserved factors for modelling different degrees of cross-sectional dependence in the errors, as measured by the maximal cross-sectional exponents of the unobserved factors.

The observations for the static panel are generated as

$$
y_{i t}=\mu_{i}+\beta_{i} x_{i t}+u_{i t}, \text { for } i=1,2, \ldots, N ; t=1,2, \ldots, T,
$$

where $\mu_{i} \sim \operatorname{IIDN}(1,1)$

$$
x_{i t}=\rho_{i x} x_{i t-1}+\nu_{i t}, i=1,2, \ldots N \text { for } t=1,2, \ldots, T,
$$

$\nu_{i t} \sim \operatorname{IIDN}(0,1)$, and $x_{i 0}=\left(1-\rho_{x}^{2}\right)^{-1 / 2} \nu_{i 0}$, for $i=1,2, \ldots N$. We do not expect the small sample properties of the CD test to depend on the nature of the regressors, and throughout the experiments we set $\rho_{i x}=0.9$. We allow for heterogeneous slopes by generating them as $\beta_{i} \sim \operatorname{IIDN}(1,1)$, for $i=1,2, \ldots, N$.

The errors, $u_{i t}$, are generated as a serially uncorrelated multi-factor process:

$$
u_{i t}=\gamma_{i 1} f_{1 t}+\gamma_{i 2} f_{2 t}+\varepsilon_{i t},
$$

with $\varepsilon_{i t} \sim \operatorname{IIDN}\left(0, \sigma_{i \varepsilon}^{2}\right), \sigma_{i \varepsilon}^{2} \sim \operatorname{IID} \chi^{2}(2) / 2$, for $i=1,2, \ldots, N$. The factors are generated as $f_{j t} \sim \operatorname{IIDN}(0,1)$, for $j=1$ and 2 . The factor loadings are generated as:

$$
\begin{aligned}
& \gamma_{j i}=v_{j i}, \text { for } i=1,2, \ldots, M_{j} \text { and } j=1,2, \\
& \gamma_{j i}=\rho_{\gamma}^{i-M_{j}}, \text { for } i=M_{j}+1, M_{j}+2, \ldots, N \text { and } j=1,2
\end{aligned}
$$

\footnotetext{
${ }^{11}$ In the more general case where the panel data model contains lagged dependent variables as well as exogenous regressors, the symmetry of error distribution does not seem to be sufficient for the symmetry of the residuals, and the problem requires further investigations.
} 
where $M_{j}=\left[N^{\alpha_{j}}\right]$ for $j=1,2, v_{j i} \sim \operatorname{IIDU}\left(\mu_{v_{j}}-0.5, \mu_{v_{j}}+0.5\right)$. We set $\mu_{v_{j}}=1$ for $j=1,2$. We set $\rho_{\gamma}=0$, since our preliminary analysis suggested that the results are not much affected by the choice of $\rho_{\gamma}$, although one would expect that the performance of the CD test to deteriorate if values of $\rho_{\gamma}$ close to unity are considered. In such cases larger sample sizes $(N)$ are needed. Here by setting $\rho_{\gamma}=0$, we are also able to consider the baseline case where the errors are cross-sectionally independent, which corresponds to $\alpha=0$ if $\rho_{\gamma}=0$. But if $\rho_{\gamma} \neq 0$ one does not obtain error cross-sectional independence by setting $\alpha=0$.

We considered a one-factor as well as a two-factor specification. In the one-factor case we set

$$
\alpha=(0,0.1,0.2,0.25,0.35,0.5,0.65,0.75,0.85,0.9,1) .
$$

In the two-factor case $\alpha_{1} \geq \alpha_{2}$, and $\alpha=\max \left(\alpha_{1}, \alpha_{2}\right)$. More specifically, we set

$$
\left(\alpha_{1}, \alpha_{2}\right)=\left[\begin{array}{c}
(0,0),(0.1,0),(0.2,0.1),(0.25,0.15),(0.35,0.25),(0.50,0.25) \\
(0.65,0.25),(0.75,0.25),(0.85,0.25),(0.90,0.25),(1.0,0.25)
\end{array}\right]
$$

so that in the case of the two-factor model we also have

$$
\alpha=\max \left(\alpha_{1}, \alpha_{2}\right)=(0,0.1,0.2,0.25,0.35,0.5,0.65,0.75,0.85,0.9,1) .
$$

The dynamic panel data model was generated as a second-order autoregressive process with heterogeneous slopes:

$$
y_{i t}=\left(1-\lambda_{i 1}-\lambda_{i 2}\right) \mu_{i}+\lambda_{i 1} y_{i, t-1}+\lambda_{i 2} y_{i, t-2}+u_{i t} .
$$

$\mu_{i}$ and $u_{i t}$ were generated exactly as in the case of the static specification. The autoregressive coefficients, $\lambda_{i 1}$ and $\lambda_{i 2}$, were generated as $\lambda_{i 1} \sim \operatorname{IIDU}(0,0.4)$, and $\lambda_{i 2}=0.2$, for all $i$, and fixed across replications.

All experiments were carried out for $N=20,50,100,250,500$ and $T=20,50,100$, to evaluate the applicability of the CD test to panels where $N$ is much larger than $T$. The number of replications was set to 2000 .

The results are summarized in Tables 1 and 2 for the static and dynamic specifications, respectively. The tables give the rejection frequencies of the $\mathrm{CD}$ test for different values of $\alpha$, sample sizes $N$ and $T$. The left panels of the tables refer to the one-factor error models and the right panels to the two-factor case. For all values of $N$ and $T$ the rejection frequencies are around $5 \%$ (the nominal size of the $C D$ test) when $\alpha<1 / 4$ and start to rise significantly as $\alpha$ approaches and exceed the 0.5 threshold, and attains its maximum of unity for $\alpha \geq 0.75$. These findings hold equally for static and dynamic models. However, at $\alpha=1 / 4$, there is some evidence of over rejection ( $7 \%$ as compared to $5 \%$ ) when $N$ is small relative to $T$, namely for $N=20$ and $T=100$.

The Monte Carlo evidence matches the asymptotic theory remarkably well, and suggests that the test can be used fruitfully as a prelude to the estimation and inference concerning the values of $\alpha$ in the range $[0.70,1]$ which are typically identified with strong factor dependence. See also Bailey, Kapetanios and Pesaran (2012).

\section{Concluding Remarks}

This paper provides a rigorous proof of the validity of the CD test proposed in Pesaran (2004), and further establishes that the CD test is best viewed as a test of weak cross-sectional dependence. 
The null hypothesis of the CD test is shown to be $\alpha<(2-\epsilon) / 4$, where $\alpha$ is the exponent of cross-sectional dependence introduced in Bailey, Kapetanios and Pesaran (2012), and $\epsilon$ measures the degree to which $T$ expands relative to $N$, as defined by $T=O\left(N^{\epsilon}\right)$, for values of $0<\epsilon \leq 1$. It is shown that the CD test is particularly powerful against $\alpha>1 / 2$, and its power rises with $\alpha$ and in $\sqrt{T} N$. As a test of weak cross-sectional dependence, the CD test continues to be valid under fairly general conditions even when $T$ is small and $N$ large. The test can be applied to balanced and unbalanced panels and is shown to have a standard normal distribution assuming that the errors are symmetrically distributed. The Monte Carlo evidence reported in the paper shows that the $C D$ statistic provides a simple and powerful test of weak cross-sectional dependence in the case of static as well as dynamic panels.

As a possible area of further research it would be interesting to investigate if the test of crosssectional independence proposed in Hsiao, Pesaran and Pick (2012) for non-linear panel data models can also be viewed as a test of weak-cross-sectional dependence, and in particular determine the range of values of $\alpha$ for which the test has power. 


\section{References}

[1] Anselin, L. (1988), Spatial Econometrics: Methods and Models, Dorddrecht: Kluwer Academic Publishers.

[2] Anselin, L. (2001), "Spatial Econometrics", in B. Baltagi (ed.), A Companion to Theoretical Econometrics, Blackwell, Oxford.

[3] Bailey, N., G. Kapetanios, and Pesaran, M.H. (2012), "Exponent of Cross-sectional Dependence: Estimation and Inference", University of Cambridge Working Papers in Economics 1206, Faculty of Economics, University of Cambridge.

[4] Baltagi, B. Q. Feng, and Kao, C. (2011), "Testing for Sphericity in a Fixed Effects Panel Data Model. The Econometrics Journal 14, 25-47.

[5] Breusch, T.S., and Pagan, A.R. (1980), "The Lagrange Multiplier Test and its Application to Model Specifications in Econometrics", Review of Economic Studies, 47, 239-53.

[6] Chamberlain, G. (1983), "Funds, factors and diversification in Arbitrage pricing theory", Econometrica 51, 1305-1323.

[7] Chudik, A., Pesaran, M. H. and Tosetti, E. (2011), "Weak and strong cross-section dependence and estimation of large panels", The Econometrics Journal, 14, C45-C90.

[8] Cliff, A. and Ord, J.K. (1973), Spatial Aurocorrection, London: Pion.

[9] Cliff, A and Ord, J.K. (1981), Spatial Processes: Models and Applications, London: Pion.

[10] Conley, T.G. and Topa, G. (2002), "Socio-economic Distance and Spatial Patterns in Unemployment", Journal of Applied Econometrics 17, 303 - 327.

[11] Frees, E. W. (1995). Assessing cross-sectional correlation in panel data. Journal of Econometrics $69,393-414$.

[12] Haining, R.P. (2003), Spatial data Analysis: Theory and Practice, Cambridge University Press, Cambridge.

[13] Hsiao, C., M.H. Pesaran, Pick, A. (2012), "Diagnostic Tests of Cross-section Independence for Limited Dependent Variable Panel Data Models", Oxford Bulletin of Economics and Statsitics, forthcoming.

[14] Lieberman, O. (1994), "A Laplace approximation to the moments of a ratio of quadratic forms", Biometrika 81, 681-690.

[15] Moscone, F. and Tosetti, E. (2009), "A Review and Comparisons of Tests of Cross-Section Independence in Panels", Journal of Economic Surveys, 23, 528-561.

[16] Moran, P.A.P. (1948), "The Interpretation of Statistical Maps", Biometrika, 35, 255-60.

[17] Pesaran, M.H. (2004), "General Diagnostic Tests for Cross Section Dependence in Panels", CESifo Working Paper Series No. 1229; IZA Discussion Paper No. 1240. Available at SSRN: http://ssrn.com/abstract $=572504$. 
[18] Pesaran, M.H., Schuermann, T., and Weiner, S.M. (2004), "Modeling Regional Interdependencies using a Global Error-Correcting Macroeconomic Model", Journal of Business Economics and Statistics (with Discussions and a Rejoinder), 22, 129-181.

[19] Pesaran, M.H., A. Ullah, and T. Yamagata, (2008), "A Bias-Adjusted LM Test Of Error Cross Section Independence", The Econometrics Journal, 11, 105-127.

[20] Sarafidis, V., T. Yamagata, D. Robertson (2009), "A Test of Cross Section Dependence for a Linear Dynamic Panel Model with Regressors", Journal of Econometrics, 148, 149-161.

[21] Sarafidis, V. and T. Wansbeek, (2012), "Cross-sectional Dependence in Panel Data Analysis", Econometric Reviews, forthcoming.

[22] Zellner, A. (1962), "An Efficient Method for Estimating Seemingly Unrelated Regressions and Tests of Aggregation Bias", Journal of American Statistical Association, 58, 977-992. 
Table 1: Rejection frequencies of the CD test at 5\% significance level for static heterogeneous panels with one exogenous regressor

\begin{tabular}{|c|c|c|c|c|c|c|c|c|c|}
\hline \multirow[b]{2}{*}{$\alpha$} & \multirow[b]{2}{*}{$\mathrm{N} \backslash \mathrm{T}=$} & \multicolumn{3}{|c|}{ One factor } & \multirow[b]{2}{*}{$\alpha$} & \multirow[b]{2}{*}{$\mathrm{N} \backslash \mathrm{T}=$} & \multicolumn{3}{|c|}{ Two factors } \\
\hline & & 20 & 50 & 100 & & & 20 & 50 & 100 \\
\hline 0.00 & 20 & 0.060 & 0.049 & 0.061 & 0.00 & 20 & 0.056 & 0.057 & 0.054 \\
\hline 0.10 & & 0.060 & 0.049 & 0.061 & 0.10 & & 0.056 & 0.057 & 0.054 \\
\hline 0.20 & & 0.060 & 0.049 & 0.061 & 0.20 & & 0.056 & 0.057 & 0.054 \\
\hline 0.25 & & 0.082 & 0.063 & 0.091 & 0.25 & & 0.071 & 0.072 & 0.080 \\
\hline 0.35 & & 0.082 & 0.063 & 0.091 & 0.35 & & 0.080 & 0.086 & 0.097 \\
\hline 0.50 & & 0.263 & 0.428 & 0.664 & 0.50 & & 0.236 & 0.364 & 0.598 \\
\hline 0.65 & & 0.831 & 0.996 & 1.000 & 0.65 & & 0.781 & 0.973 & 1.000 \\
\hline 0.75 & & 0.988 & 1.000 & 1.000 & 0.75 & & 0.981 & 0.999 & 1.000 \\
\hline 0.85 & & 1.000 & 1.000 & 1.000 & 0.85 & & 1.000 & 1.000 & 1.000 \\
\hline 0.90 & & 1.000 & 1.000 & 1.000 & 0.90 & & 1.000 & 1.000 & 1.000 \\
\hline 1.00 & & 1.000 & 1.000 & 1.000 & 1.00 & & 1.000 & 1.000 & 1.000 \\
\hline 0.00 & 50 & 0.059 & 0.049 & 0.055 & 0.00 & 50 & 0.063 & 0.051 & 0.055 \\
\hline 0.10 & & 0.059 & 0.049 & 0.055 & 0.10 & & 0.063 & 0.051 & 0.055 \\
\hline 0.20 & & 0.069 & 0.054 & 0.067 & 0.20 & & 0.074 & 0.060 & 0.056 \\
\hline 0.25 & & 0.069 & 0.054 & 0.067 & 0.25 & & 0.074 & 0.060 & 0.056 \\
\hline 0.35 & & 0.083 & 0.074 & 0.096 & 0.35 & & 0.085 & 0.078 & 0.086 \\
\hline 0.50 & & 0.383 & 0.546 & 0.756 & 0.50 & & 0.297 & 0.576 & 0.716 \\
\hline 0.65 & & 0.907 & 0.997 & 1.000 & 0.65 & & 0.883 & 0.995 & 1.000 \\
\hline 0.75 & & 1.000 & 1.000 & 1.000 & 0.75 & & 1.000 & 1.000 & 1.000 \\
\hline 0.85 & & 1.000 & 1.000 & 1.000 & 0.85 & & 1.000 & 1.000 & 1.000 \\
\hline 0.90 & & 1.000 & 1.000 & 1.000 & 0.90 & & 1.000 & 1.000 & 1.000 \\
\hline 1.00 & & 1.000 & 1.000 & 1.000 & 1.00 & & 1.000 & 1.000 & 1.000 \\
\hline 0.00 & 100 & 0.062 & 0.056 & 0.061 & 0.00 & 100 & 0.048 & 0.048 & 0.049 \\
\hline 0.10 & & 0.062 & 0.056 & 0.061 & 0.10 & & 0.048 & 0.048 & 0.049 \\
\hline 0.20 & & 0.064 & 0.062 & 0.066 & 0.20 & & 0.054 & 0.054 & 0.052 \\
\hline 0.25 & & 0.069 & 0.071 & 0.079 & 0.25 & & 0.054 & 0.060 & 0.060 \\
\hline 0.35 & & 0.094 & 0.125 & 0.178 & 0.35 & & 0.078 & 0.107 & 0.135 \\
\hline 0.50 & & 0.306 & 0.646 & 0.886 & 0.50 & & 0.302 & 0.647 & 0.774 \\
\hline 0.65 & & 0.955 & 1.000 & 1.000 & 0.65 & & 0.960 & 1.000 & 1.000 \\
\hline 0.75 & & 1.000 & 1.000 & 1.000 & 0.75 & & 1.000 & 1.000 & 1.000 \\
\hline 0.85 & & 1.000 & 1.000 & 1.000 & 0.85 & & 1.000 & 1.000 & 1.000 \\
\hline 0.90 & & 1.000 & 1.000 & 1.000 & 0.90 & & 1.000 & 1.000 & 1.000 \\
\hline 1.00 & & 1.000 & 1.000 & 1.000 & 1.00 & & 1.000 & 1.000 & 1.000 \\
\hline 0.00 & 250 & 0.059 & 0.049 & 0.050 & 0.00 & 250 & 0.055 & 0.052 & 0.052 \\
\hline 0.10 & & 0.059 & 0.049 & 0.050 & 0.10 & & 0.055 & 0.052 & 0.052 \\
\hline 0.20 & & 0.061 & 0.052 & 0.053 & 0.20 & & 0.061 & 0.058 & 0.056 \\
\hline 0.25 & & 0.061 & 0.052 & 0.053 & 0.25 & & 0.059 & 0.059 & 0.055 \\
\hline 0.35 & & 0.085 & 0.084 & 0.090 & 0.35 & & 0.075 & 0.079 & 0.095 \\
\hline 0.50 & & 0.317 & 0.541 & 0.816 & 0.50 & & 0.302 & 0.464 & 0.841 \\
\hline 0.65 & & 0.994 & 1.000 & 1.000 & 0.65 & & 0.996 & 1.000 & 1.000 \\
\hline 0.75 & & 1.000 & 1.000 & 1.000 & 0.75 & & 1.000 & 1.000 & 1.000 \\
\hline 0.85 & & 1.000 & 1.000 & 1.000 & 0.85 & & 1.000 & 1.000 & 1.000 \\
\hline 0.90 & & 1.000 & 1.000 & 1.000 & 0.90 & & 1.000 & 1.000 & 1.000 \\
\hline 1.00 & & 1.000 & 1.000 & 1.000 & 1.00 & & 1.000 & 1.000 & 1.000 \\
\hline 0.00 & 500 & 0.054 & 0.052 & 0.050 & 0.00 & 500 & 0.061 & 0.060 & 0.058 \\
\hline 0.10 & & 0.054 & 0.052 & 0.050 & 0.10 & & 0.061 & 0.060 & 0.058 \\
\hline 0.20 & & 0.056 & 0.053 & 0.047 & 0.20 & & 0.062 & 0.055 & 0.058 \\
\hline 0.25 & & 0.056 & 0.053 & 0.050 & 0.25 & & 0.064 & 0.064 & 0.063 \\
\hline 0.35 & & 0.074 & 0.081 & 0.087 & 0.35 & & 0.081 & 0.091 & 0.091 \\
\hline 0.50 & & 0.402 & 0.690 & 0.849 & 0.50 & & 0.350 & 0.622 & 0.827 \\
\hline 0.65 & & 1.000 & 1.000 & 1.000 & 0.65 & & 1.000 & 1.000 & 1.000 \\
\hline 0.75 & & 1.000 & 1.000 & 1.000 & 0.75 & & 1.000 & 1.000 & 1.000 \\
\hline 0.85 & & 1.000 & 1.000 & 1.000 & 0.85 & & 1.000 & 1.000 & 1.000 \\
\hline 0.90 & & 1.000 & 1.000 & 1.000 & 0.90 & & 1.000 & 1.000 & 1.000 \\
\hline 1.00 & & 1.000 & 1.000 & 1.000 & 1.00 & & 1.000 & 1.000 & 1.000 \\
\hline
\end{tabular}


Table 2: Rejection frequencies of the CD test at 5\% significance level for $\operatorname{AR}(2)$ heterogeneous panels

\begin{tabular}{|c|c|c|c|c|c|c|c|c|c|}
\hline \multicolumn{5}{|c|}{ One factor } & \multicolumn{5}{|c|}{ Two factors } \\
\hline$\alpha$ & $\mathrm{N} \backslash \mathrm{T}=$ & 20 & 50 & 100 & $\alpha$ & $\mathrm{N} \backslash \mathrm{T}=$ & 20 & 50 & 100 \\
\hline 0.00 & 20 & 0.058 & 0.052 & 0.050 & 0.00 & 20 & 0.051 & 0.047 & 0.056 \\
\hline 0.10 & & 0.058 & 0.052 & 0.050 & 0.10 & & 0.051 & 0.047 & 0.056 \\
\hline 0.20 & & 0.058 & 0.052 & 0.050 & 0.20 & & 0.051 & 0.047 & 0.056 \\
\hline 0.25 & & 0.070 & 0.072 & 0.076 & 0.25 & & 0.059 & 0.071 & 0.088 \\
\hline 0.35 & & 0.070 & 0.072 & 0.076 & 0.35 & & 0.064 & 0.078 & 0.115 \\
\hline 0.50 & & 0.213 & 0.370 & 0.577 & 0.50 & & 0.188 & 0.345 & 0.583 \\
\hline 0.65 & & 0.809 & 0.993 & 0.999 & 0.65 & & 0.758 & 0.979 & 0.999 \\
\hline 0.75 & & 0.977 & 1.000 & 1.000 & 0.75 & & 0.971 & 1.000 & 1.000 \\
\hline 0.85 & & 0.999 & 1.000 & 1.000 & 0.85 & & 0.999 & 1.000 & 1.000 \\
\hline 0.90 & & 1.000 & 1.000 & 1.000 & 0.90 & & 1.000 & 1.000 & 1.000 \\
\hline 1.00 & & 1.000 & 1.000 & 1.000 & 1.00 & & 1.000 & 1.000 & 1.000 \\
\hline 0.00 & 50 & 0.055 & 0.045 & 0.049 & 0.00 & 50 & 0.047 & 0.051 & 0.055 \\
\hline 0.10 & & 0.055 & 0.045 & 0.049 & 0.10 & & 0.047 & 0.051 & 0.055 \\
\hline 0.20 & & 0.059 & 0.052 & 0.057 & 0.20 & & 0.049 & 0.054 & 0.061 \\
\hline 0.25 & & 0.059 & 0.052 & 0.057 & 0.25 & & 0.049 & 0.054 & 0.061 \\
\hline 0.35 & & 0.080 & 0.070 & 0.087 & 0.35 & & 0.062 & 0.076 & 0.092 \\
\hline 0.50 & & 0.261 & 0.599 & 0.771 & 0.50 & & 0.217 & 0.492 & 0.750 \\
\hline 0.65 & & 0.815 & 0.997 & 1.000 & 0.65 & & 0.758 & 0.996 & 1.000 \\
\hline 0.75 & & 0.997 & 1.000 & 1.000 & 0.75 & & 0.995 & 1.000 & 1.000 \\
\hline 0.85 & & 1.000 & 1.000 & 1.000 & 0.85 & & 1.000 & 1.000 & 1.000 \\
\hline 0.90 & & 1.000 & 1.000 & 1.000 & 0.90 & & 1.000 & 1.000 & 1.000 \\
\hline 1.00 & & 1.000 & 1.000 & 1.000 & 1.00 & & 1.000 & 1.000 & 1.000 \\
\hline 0.00 & 100 & 0.059 & 0.046 & 0.051 & 0.00 & 100 & 0.054 & 0.050 & 0.054 \\
\hline 0.10 & & 0.059 & 0.046 & 0.051 & 0.10 & & 0.054 & 0.050 & 0.054 \\
\hline 0.20 & & 0.065 & 0.050 & 0.051 & 0.20 & & 0.059 & 0.054 & 0.053 \\
\hline 0.25 & & 0.067 & 0.051 & 0.061 & 0.25 & & 0.068 & 0.067 & 0.066 \\
\hline 0.35 & & 0.098 & 0.089 & 0.139 & 0.35 & & 0.094 & 0.113 & 0.155 \\
\hline 0.50 & & 0.319 & 0.523 & 0.860 & 0.50 & & 0.264 & 0.610 & 0.867 \\
\hline 0.65 & & 0.957 & 1.000 & 1.000 & 0.65 & & 0.866 & 1.000 & 1.000 \\
\hline 0.75 & & 1.000 & 1.000 & 1.000 & 0.75 & & 1.000 & 1.000 & 1.000 \\
\hline 0.85 & & 1.000 & 1.000 & 1.000 & 0.85 & & 1.000 & 1.000 & 1.000 \\
\hline 0.90 & & 1.000 & 1.000 & 1.000 & 0.90 & & 1.000 & 1.000 & 1.000 \\
\hline 1.00 & & 1.000 & 1.000 & 1.000 & 1.00 & & 1.000 & 1.000 & 1.000 \\
\hline 0.00 & 250 & 0.060 & 0.047 & 0.044 & 0.00 & 250 & 0.056 & 0.048 & 0.052 \\
\hline 0.10 & & 0.060 & 0.047 & 0.044 & 0.10 & & 0.056 & 0.048 & 0.052 \\
\hline 0.20 & & 0.057 & 0.053 & 0.047 & 0.20 & & 0.059 & 0.055 & 0.055 \\
\hline 0.25 & & 0.057 & 0.053 & 0.047 & 0.25 & & 0.059 & 0.052 & 0.055 \\
\hline 0.35 & & 0.076 & 0.081 & 0.088 & 0.35 & & 0.071 & 0.077 & 0.089 \\
\hline 0.50 & & 0.311 & 0.577 & 0.808 & 0.50 & & 0.254 & 0.525 & 0.851 \\
\hline 0.65 & & 0.996 & 1.000 & 1.000 & 0.65 & & 0.992 & 1.000 & 1.000 \\
\hline 0.75 & & 1.000 & 1.000 & 1.000 & 0.75 & & 1.000 & 1.000 & 1.000 \\
\hline 0.85 & & 1.000 & 1.000 & 1.000 & 0.85 & & 1.000 & 1.000 & 1.000 \\
\hline 0.90 & & 1.000 & 1.000 & 1.000 & 0.90 & & 1.000 & 1.000 & 1.000 \\
\hline 1.00 & & 1.000 & 1.000 & 1.000 & 1.00 & & 1.000 & 1.000 & 1.000 \\
\hline 0.00 & 500 & 0.050 & 0.047 & 0.053 & 0.00 & 500 & 0.049 & 0.050 & 0.050 \\
\hline 0.10 & & 0.050 & 0.047 & 0.053 & 0.10 & & 0.049 & 0.050 & 0.050 \\
\hline 0.20 & & 0.052 & 0.046 & 0.056 & 0.20 & & 0.049 & 0.050 & 0.053 \\
\hline 0.25 & & 0.058 & 0.047 & 0.060 & 0.25 & & 0.050 & 0.052 & 0.055 \\
\hline 0.35 & & 0.066 & 0.077 & 0.099 & 0.35 & & 0.064 & 0.069 & 0.083 \\
\hline 0.50 & & 0.308 & 0.571 & 0.803 & 0.50 & & 0.313 & 0.533 & 0.789 \\
\hline 0.65 & & 0.998 & 1.000 & 1.000 & 0.65 & & 0.998 & 1.000 & 1.000 \\
\hline 0.75 & & 1.000 & 1.000 & 1.000 & 0.75 & & 1.000 & 1.000 & 1.000 \\
\hline 0.85 & & 1.000 & 1.000 & 1.000 & 0.85 & & 1.000 & 1.000 & 1.000 \\
\hline 0.90 & & 1.000 & 1.000 & 1.000 & 0.90 & & 1.000 & 1.000 & 1.000 \\
\hline 1.00 & & 1.000 & 1.000 & 1.000 & 1.00 & & 1.000 & 1.000 & 1.000 \\
\hline
\end{tabular}

